\title{
Optimal Systematic Determination of Models' Base for Multimodel Representation: Real Time Application
}

\author{
Majda Ltaief Anis Messaoud Ridha Ben Abdennour \\ Research Unit of Numerical Control of Industrial Processes, National School of Engineers of Gabes, University of Gabes St Omar Ibn \\ El-Khattab, 6029 Gabes, Tunisia
}

\begin{abstract}
The multimodel approach is a powerful and practical tool to deal with analysis, modeling, observation, emulation and control of complex systems. In the modeling framework, we propose in this paper a new method for optimal systematic determination of models' base for multimodel representation. This method is based on the classification of data set picked out of the considered system. The obtained cluster centers are exploited to provide the weighting functions and to deduce the corresponding dispersions and their models' base. A simulation example and an experimental validation on a semi-batch reactor are presented to evaluate the effectiveness of the proposed method.
\end{abstract}

Keywords: Multimodel approach, identification, classification, weighting functions, complex systems, chemical reactor.

\section{Introduction}

The multimodel approach is an interesting alternative and a powerful tool for representation, observation, emulation and control of complex processes.

The basic idea of this approach is the decomposition of the full operation range of the process into a number of operating regimes. In each operating regime, a simple local model is considered. These local models called model's library are then combined in some way to yield a global model.

Nonlinear models are also widely used in engineering science applications to describe the dynamic behavior of real processes. Due to their mathematical complexity, they are not easily exploitable for designing an observer, an emulator, a control law or for setting up a diagnosis strategy, even if they give a good description of the considered process. Assuming that the modeled process evolves around an operating point, a linearization procedure is then possible and leads to the reduction of the mathematical complexity of the nonlinear model. Hence, analysis tools available for linear systems can be used. However, in practical cases, the linearity assumption is not always valid and consequently the linearized model does not represent the whole behavior of the process ${ }^{[1,2]}$.

Moreover, it will be more interesting in practice to have a model that is easily usable and gives a good global characterization of the process dynamic behavior.

Different approaches exist in the literature dealing with the multimodel representation. Initially, the founder of the multimodel approach focused on the regime decomposition and the choice of local model structure on the base of a combination of qualitative, quantitative, empirical and mechanistic system knowledge ${ }^{[3-5]}$. A fuzzy set methods for local modeling and identification of nonlinear systems were described in [1]. In which, local models structure was

Regular paper

Manuscript received April 4, 2013; accepted November 21, 2013 described by means of fuzzy "if-then" rules and any prior knowledge about the operating regime was not required. Other approaches based on the Takagi-Sugeno model were developed in $[6,7]$. For the representation of discrete and continuous linear systems with uncertain parameters, some methods related to the systematic generation of a models ${ }^{\prime}$ base were proposed in [8-13].

Two basic multimodel structures for interconnecting the sub-models (local models) can be distinguished ${ }^{[14]}$.

1) In the first structure, the sub-models have the same state vector (Takagi-Sugeno multimodels). This leads to the same structure for all sub-models. Which limits the choice of the models' base. Several works dealing with modeling, state estimation, control and diagnosis of nonlinear systems were based on this multimodel structure ${ }^{[7,15-18]}$.

2) In the second one, the sub-models are uncoupled and their state vectors are different (uncoupled multimodel). So we can consider sub-models with different structures. As works dealing with this multimodel structure, we cite [1923].

In the two aforementioned multimodel structures, the contribution of the sub-models depends on the weighting functions. The modeling performances are closely related to the choice of the centers and degrees of overlap between them (for example, the dispersions for Gaussian functions case). In this frame work, we propose a new method to optimize these parameters for the systematic generation of weighting function centers and dispersions. This method integrates a classification procedure of an identification data set picked out of the considered system.

The paper is organized as follows: We present in Section 2 , the principle of the multimodel representation. The proposed method for an optimal systematic generation of the weighting function centers and dispersions is detailed in Section 3. The next section presents the results of a numerical simulation example. An experimental validation on a semi-batch reactor is given in Section 5. Conclusions will 
be drawn in Section 6 .

\section{Multimodel representation}

"Divide and conquer" strategy is the basic idea of the multimodel approach. It consists in subdividing the full operating range of the system into several operating zones. At each operating zone a simple linear sub-model is identified using the known tools of linear systems identification, linearization techniques or other techniques. The global model, which is valid under all operating conditions of the system, is thereafter formed by a fusion of the sub-models and it is easily exploited to study any local proprieties and to deduce simple and powerful observers, emulators and controls. These sub-models are typical, but not always selected. The multimodel output (the output of the global model) is generated through an interpolation mechanism that integrates an online weighting functions evaluation.

\subsection{Weighting functions}

The sub-models contributions can be quantified by the weighting functions $\mu_{i}(\xi(k))$ that ensure smooth transition between sub-models. It can be obtain, for example, from the normalized Gaussian function:

$$
\mu_{i}(\xi(k))=\frac{\omega_{i}(\xi(k))}{\sum_{j=1}^{N} \omega_{j}(\xi(k))}
$$

where

$$
\omega_{i}(\xi(k))=\exp \left(-\frac{\left(\xi(k)-c_{i}\right)^{2}}{\sigma_{i}^{2}}\right)
$$

where $c_{i}$ is the centre of the $i$-th weighting function and $\sigma_{i}$ is the dispersion for weighting functions, $N$ is the number of models. The weighting functions satisfy the following constraint $^{[1,3,4,19-22,24]}$ :

$$
\begin{aligned}
& \sum_{i=1}^{N} \mu_{i}(\xi(k))=1, \quad \forall k \\
& 0 \leqslant \mu_{i}(\xi(k)) \leqslant 1, \quad \forall k, \quad \forall i=1, \cdots, N
\end{aligned}
$$

where $\xi(k)$ is the decision variable. It can be an exogenous measured signals: for example, input, output and state variable. A large choice of weighting functions is possible such as Gaussian functions, sigmoidal functions, etc. There are various ways of connecting sub-models in order to generate the multimodel output $y_{M M}(k)$. We distinguish two multimodel structures according to the use of coupled or uncoupled states.

\section{$2.2 \quad$ State multimodel structure}

Two multimodel structures are possible. The coupled state multimodel structure also called Takagi-Sugeno multimodel ${ }^{[3,4,24]}$ is extensively used in the multimodel analysis and synthesis. This multimodel structure is given by

$$
\begin{aligned}
& x(k+1)=\sum_{i=1}^{N} \mu_{i}(\xi(k))\left\{A_{i} x(k)+B_{i} u(k)\right\} \\
& y_{M M}(k)=\sum_{i=1}^{N} \mu_{i}(\xi(k)) C_{i} x(k)
\end{aligned}
$$

where $x \in \mathbf{R}^{n}$ is the common state vector of the sub-models, $u \in \mathbf{R}^{m}$ the input, $y_{M M} \in \mathbf{R}^{p}$ is the multimodel output, $A_{i} \in \mathbf{R}^{n \times n}, B_{i} \in \mathbf{R}^{n \times m}$ and $C_{i} \in \mathbf{R}^{p \times n}$ are constant matrices. In this multimodel framework, the contribution of each sub-model is evaluated through a weighted sum of submodels parameters. Therefore, this multimodel structure can be considered as a system with time varying parameters (we note that there is only a global state $x_{M M}$ ) and we have

$$
\begin{aligned}
x(k+1) & =\widetilde{A}(k) x(k)+\widetilde{B}(k) u(k) \\
y_{M M}(k) & =\widetilde{C}(k) x(k)
\end{aligned}
$$

where $\widetilde{A}(k)=\sum_{i=1}^{N} \mu_{i}(\xi(k)) A_{i}, \widetilde{B}(k)=\sum_{i=1}^{N} \mu_{i}(\xi(k)) B_{i}$ and $\widetilde{C}(k)=\sum_{i=1}^{N} \mu_{i}(\xi(k)) C_{i}$.

The other possible structure is called the uncoupled state multimodel ${ }^{[14,19-22]}$ :

$$
\begin{aligned}
& x_{i}(k+1)=A_{i}(k) x_{i}(k)+B_{i}(k) u(k) \\
& y_{i}(k)=C_{i}(k) x_{i}(k)
\end{aligned}
$$

and

$$
y_{M M}(k)=\sum_{i=1}^{N} \mu_{i}(\xi(k)) y_{i}(k)
$$

where $x_{i} \in \mathbf{R}^{n_{i}}$ and $y_{i} \in \mathbf{R}^{p}$ are respectively the state vector and the output vector of the $i$-th sub-model, $u \in \mathbf{R}^{m}$ is the input, $y_{M M} \in \mathbf{R}^{p}$ is the multimodel output, $A_{i} \in$ $\mathbf{R}^{n_{i} \times n_{i}}, B_{i} \in \mathbf{R}^{n_{i} \times m}$ and $C_{i} \in \mathbf{R}^{p \times n_{i}}$ are constant matrices. In this case the multimodel output is formulated by a weighted sum of sub-models outputs. Therefore, each sub-model evolves independently in its own state space depending on the input control and its initial state. The main interest of the uncoupled state multimodel structure is the decoupling between the sub-models. Indeed, different submodels structure can be used.

\subsection{Parametric estimation}

Essentially, there are two estimation criteria can be used in the parameter identification of the uncoupled multimodel: global and local criteria ${ }^{[25]}$. The retained estimation criterion, is the global one defined by

$$
J=\frac{1}{2} \sum_{k=1}^{N_{H}}\left(y_{M M}(k)-y(k)\right)^{2}
$$

where $N_{H}$ is the number of training data and $y(k)$ is the real output of the nonlinear system.

This criterion favors a good global characterization between global behavior of the nonlinear system and the multimodel. In this section, we present the parameter estimation procedure based on the uncoupled state multimodel 
approach. The column vector $\theta$ is the vector of unknown parameters of the multimodel.

$$
\theta=\left[\begin{array}{lllll}
\theta_{1}^{\mathrm{T}} & \cdots & \theta_{i}^{\mathrm{T}} \cdots \theta_{N}^{\mathrm{T}}
\end{array}\right]^{\mathrm{T}}
$$

where each column block $\theta_{i}$ is formed by the $q_{i}$ unknown parameters of the particular sub-model denoting the unknown scalar parameter of the sub-model $i$.

$$
\theta_{i}=\left[\theta_{i, 1} \cdots \theta_{i, q} \cdots \theta_{i, q_{i}}\right]^{\mathrm{T}}
$$

where $\theta_{i, q}$ denotes the unknown scalar parameter of the sub-model $i$.

The parametric estimation of $\theta$ is based on an iterative minimization procedure of a quadratic criterion $J(\theta)$ which can be a Levenberg-Marquardt's algorithm ${ }^{[26]}$ considering the persistent excitation condition.

$$
\theta(i t+1)=\theta(i t)-\Delta(i t)(H(\theta)+\lambda(i t) I)^{-1} G(\theta)
$$

where $\theta(i t)$ is the vector of parameters at a particular iteration it, $H(\theta)$ is Hessian matrix, and $G(\theta)$ is the gradient vector. The calculus of the gradient vector and the Hessian matrix are based on the calculation of sensitivity functions with respect to sub-models parameters.

The gradient $G$ is given by the simple derivation of the global criterion with respect to parameters $\theta$ as

$$
G=\frac{\partial J}{\partial \theta}=\sum_{k=1}^{N_{H}} \varepsilon(k) \frac{\partial y_{M M}(k)}{\partial \theta}
$$

where

$$
\varepsilon(k)=\left(y_{M M}(k)-y(k)\right) .
$$

Hessian matrix $H$ is obtained by a double derivation of the global criterion with respect to parameters $\theta$ as

$$
H=\sum_{k=1}^{N_{H}} \frac{\partial y_{M M}(k)}{\partial \theta} \frac{\partial y_{M M}(k)}{\partial \theta^{\mathrm{T}}}
$$

where

$$
\frac{\partial y_{M M}(k)}{\partial \theta}=\sum_{i=1}^{N} \mu_{i}(\xi(k)) \frac{\partial y_{i}(k)}{\partial \theta} .
$$

\section{Optimal systematic generation of weighting function centers}

The multimodel representation is composed of a set of sub-models. Each one is valid in a specified operating zone. The local validity of a sub-model is defined by a weighting function which tends to one in the corresponding operating zone and tends to zero outside it. The weighting function related to each sub-model can be defined in classical cases by the static characteristic or by linearization procedure. Also, they can be defined systematically using clustering techniques and fuzzy clustering techniques. The difficulties related to these last solutions are the determination of the number of clusters and their centers which will be the weighting functions centers. In this work, we propose a method for the optimal determination of the weighting functions centers based on Chiu's classification method ${ }^{[9,27,28]}$. Here, the normalized Gaussian functions is retained as

$$
\omega_{i}(\xi(k))=\exp \left(-\frac{(\xi(k)-c i)^{2}}{\sigma_{i}{ }^{2}}\right)
$$

and

$$
\mu_{i}(\xi(k))=\frac{\omega_{i}(\xi(k))}{\sum_{j=1}^{N} \omega_{j}(\xi(k))}
$$

where $c_{i}$ is the center of the $i$-th weighting function, $\sigma_{i}$ is the corresponding dispersion, and $\xi$ is the decision variable. The choice of centers $c_{i}$ has a great influence on the modeling precision.

\subsection{Influence of centers $c_{i}$ choice}

To demonstrate the influence of the centers, we choose them based on the system static characteristic. Indeed, after picking up the nonlinear system static characteristic, we proceed to subdivide it into linear zones. Each linear zone which will be represented by a sub-model is associated with a weighting function centered on the number $c_{i}$. This center is also chosen based on the system static characteristic.

\subsubsection{Identification example}

Let us use the uncoupled multimodel structure to represent the behavior of the nonlinear system ${ }^{[29]}$ :

$$
y(k+1)=\frac{y(k)}{1+y^{2}(k)}+u^{3}(k), \quad u \in\left[\begin{array}{ll}
-1 & 1
\end{array}\right]
$$

Here, the identification of the multimodel is realized by a global criterion. The weighting functions $\mu_{i}$ depend on the input signal $\xi(k)=u(k)$, and the centers are $c_{1}=$ $-0.3, c_{2}=0.0, c_{3}=0.3$ and the dispersion $\sigma=0.1$. Fig. 1 shows the resulting weighting functions evolutions. A set of input/output data points is used to build the multimodel. Fig. 2 shows the evolution of this data set). In this case, the signal used to validate the uncoupled state multimodel is given by

$$
u(k)=0.7 \sin \left(\frac{2 \pi k}{100}\right) .
$$

The resulting local models, by application of the identification algorithm, are described by the following expressions:

Sub-model 1.

$$
A_{1}=0.8909, B_{1}=0.1308, C_{1}=1 .
$$

Sub-model 2.

$$
\begin{aligned}
& A_{2}=\left[\begin{array}{cc}
0.0911 & -0.0943 \\
-0.14 & 0.9
\end{array}\right], B_{2}=\left[\begin{array}{c}
-0.3664 \\
-0.67
\end{array}\right], \\
& C_{2}=\left[\begin{array}{ll}
1 & 0
\end{array}\right] .
\end{aligned}
$$

Sub-model 3.

$$
A_{3}=0.8919, B_{3}=0.1357, C_{3}=1 .
$$

The validation results are given in Fig. 3. This figure shows the evolutions of the real and the multimodel outputs 
$\left(y(k)\right.$ and $\left.y_{M M}(k)\right)$. The simulation results show that the multimodel output cannot describe the real behavior with satisfactory precision. The mean square error (MSE) and the variance-accounted-for (VAF) have been calculated to evaluate the performance by the following equations:

$$
\begin{aligned}
& \mathrm{MSE}=\frac{1}{N_{H}} \sum_{k=1}^{N_{H}}\left(y_{M M}(k)-y(k)\right)^{2} \\
& \mathrm{VAF}=\max \left\{1-\frac{\operatorname{var}\left(y_{M M}(k)-y(k)\right)}{\operatorname{var}\left(y_{M M}(k)\right)}, 0\right\} \times 100 \% .
\end{aligned}
$$

They are respectively equal, in this case, to 0.021 and $92.17 \%$.

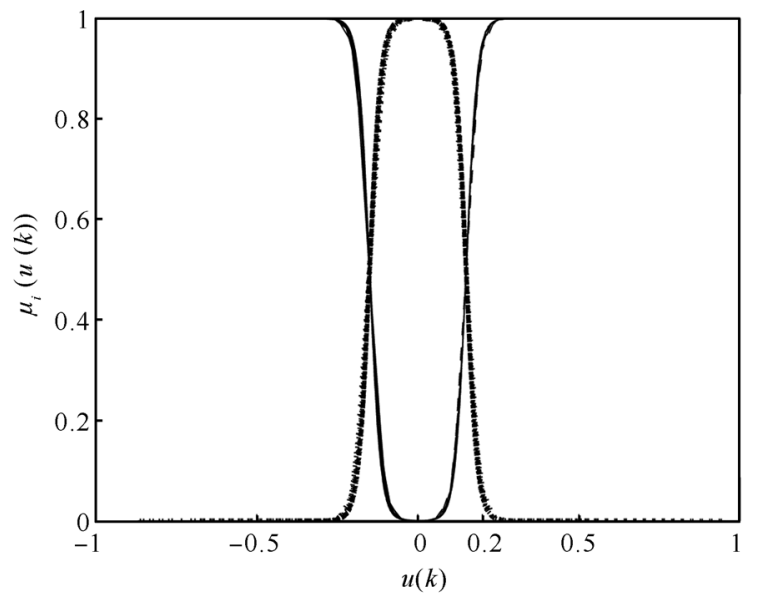

Fig. 1 Weighting functions evolutions (classical choice based on the static characteristic)
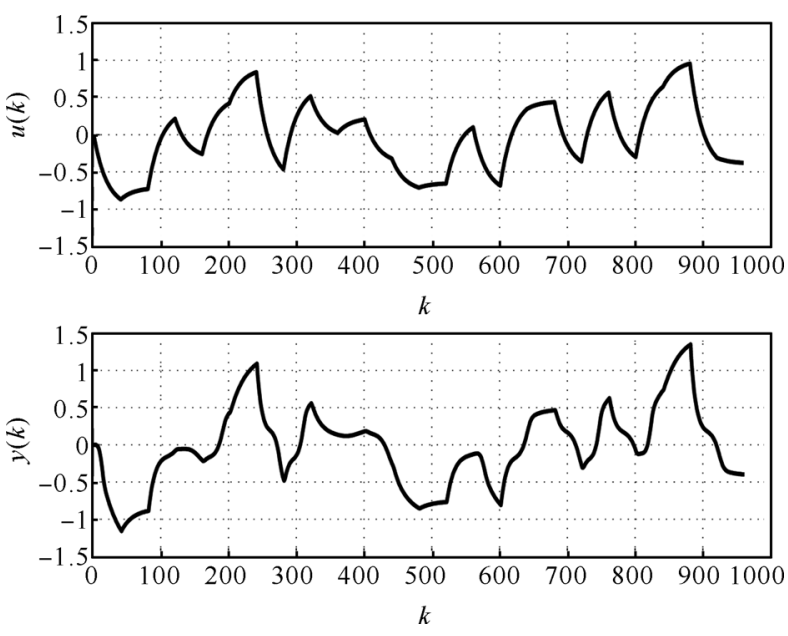

Fig. 2 Data set for system identification

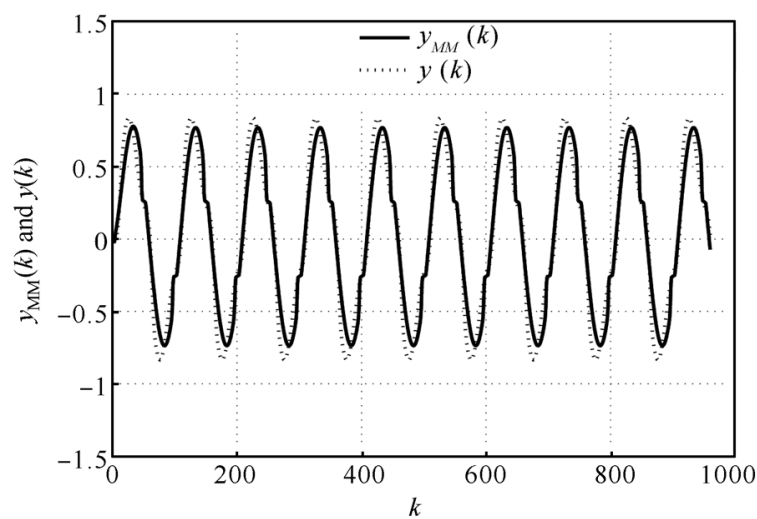

Fig. 3 Evolutions of the real and the multimodel outputs

\subsection{Proposed optimal systematic genera- tion of centers $c_{i}$}

We propose in this work to generate systematically the synthesis parameters (centers and dispersions) related to the weighting functions. Indeed, the full operating range of the nonlinear system is partitioned into a set of operating zones via a classification procedure which provides the cluster centers that will be considered as the weighting function centers. The dispersion of each weighting function is then given in function of the distances separating the neighbor centers.

\subsubsection{Classification procedure}

Having a set of numerical data, the classification procedure consists in selecting between these data, the cluster centers using Chiu's method for the numerical data classification $^{[9,27,28]}$. In this work, we aimed to extend this method to classify a set of regression vectors $\varphi_{j}\left(\varphi_{j}=\right.$ $\left.[y(j) y(j-1) u(j-1)]^{\mathrm{T}}, j=1, \cdots, M\right)$ built from a set of persistent identification data covering the hole operating range of the considered nonlinear system.

Each regression vector $\varphi_{j}$, is associated with a potential $P_{j}$ given by the expression as

$$
P_{j}=\sum_{h=1}^{M} \mathrm{e}^{\left(\frac{-4\left\|\varphi_{j}-\varphi_{h}\right\|^{2}}{r_{a}^{2}}\right)}
$$

where $r_{a}$ is a positive parameter controlling the decrease ratio of the potential. The potential decreases exponentially as $\varphi_{h}$ moves away from $\varphi_{j}$. The first regression vector cluster center $\varphi_{1}^{*}$ is the vector whose potential $P_{1}^{*}$ given by (19) is the maximum.

To avoid selecting the first cluster center $\varphi_{1}^{*}$ having the potential $P_{1}^{*}$ and its neighbors as other cluster centers, the classification procedure assigns to each potential $P_{j}$ the following new value:

$$
P_{j} \leftarrow P_{j}-P_{1}^{*} \mathrm{e}^{\left(\frac{-4\left\|\varphi_{j}-\varphi_{1}^{*}\right\|^{2}}{r_{b}^{2}}\right) .} .
$$

The parameter $r_{b}\left(r_{b}>0\right)$ must be selected larger than $r_{a}$ to favor the operation related to the selection of other cluster center completely different from the last one. Next, we select, as second cluster-center, the vector whose modified potential given by the relation (20) is the maximum. 
Let the second cluster-center be $\varphi_{2}^{*}$ and the associated potential value be $P_{2}^{*}$.

In a similar manner, we choose the $i$-th cluster center $\varphi_{i}^{*}$ with the maximum potential $P_{i}^{*}$ and modify the potentials as follows:

$$
P_{j} \leftarrow P_{j}-P_{i}^{*} \mathrm{e}^{\left(\frac{-4\left\|\varphi_{j}-\varphi_{i}^{*}\right\|^{2}}{r_{b}^{2}}\right)} .
$$

Chiu determined the cluster selection by introducing two positive parameters $\varepsilon_{1}$ and $\varepsilon_{2}\left(\varepsilon_{1}>\varepsilon_{2}\right)$. He proposed the following inequalities for stopping the cluster centers' selection.

1) If $P_{i}^{*}>\varepsilon_{1} P_{1}^{*} \Rightarrow$ Selection sanctioned.

2) If $P_{i}^{*}<\varepsilon_{2} P_{1}^{*} \Rightarrow$ Selection stopped.

3) If $\varepsilon_{2} P_{1}^{*} \leqslant P_{i}^{*} \leqslant \varepsilon_{1} P_{1}^{*}$ and if :

$$
\frac{\min \left(\min \left(\left|\varphi_{i}^{*}-\varphi_{1}^{*}\right|\right), \cdots, \min \left(\left|\varphi_{i}^{*}-\varphi_{i-1}^{*}\right|\right)\right)}{r_{a}}<1-\frac{P_{i}^{*}}{P_{1}^{*}}
$$

where $\varphi_{i}^{*}$ is the current regression vector center and $\varphi_{1}^{*}$, $\varphi_{2}^{*}, \cdots, \varphi_{i-1}^{*}$ are the last selected ones. The regression vector center to be retained corresponds, in this case, to the maximum value of the potentials after rejecting the current value $P_{i}^{*}$.

\subsubsection{Centers determination}

Once the regression vector cluster centers are selected and referring to the decision variable to be considered $(\xi(k)=y(k)$ or $\xi(k)=u(k))$, we have the weighting functions centers $c_{i}$.

$$
c_{i}=u_{i}^{*} \text { or } c_{i}=y_{i}^{*}, i=1, \cdots, N .
$$

\subsubsection{Dispersions computation}

Then, we calculate the corresponding dispersion $\sigma_{i}$ using one expression of the two given below:

Expression 1. The dispersions $\sigma_{i}$ can be adjusted according to the mean distance to the $n$ nearest neighbors ${ }^{[24]}$.

$$
\sigma_{i}=\alpha \frac{1}{n} \sum_{j=1}^{n}\left|c_{i}-c_{j}\right|
$$

where $c_{i}$ is the current center and $c_{j}$ is the nearest neighbor center to $c_{i} . \quad \alpha$ is a scaling factor defining the degree of overlap between the weighting functions.

Expression 2. The dispersions $\sigma_{i}$ is considered to be in proportion to the distance separating the center $c_{i}$ and the nearest center ${ }^{[28]}$.

$$
\sigma_{i}=\frac{1}{\alpha} \min _{j=1, \cdots, n}\left(\left|c_{i}-c_{j}\right|\right)
$$

where $\alpha$ is a positive factor defining the degree of overlap between the weighting functions. Order estimation of retained models can be insured by instrumental determinants' ratio-test method ${ }^{[2]}$.

\section{Simulation results}

We consider the same nonlinear system given by (15). The same identification data set (see Fig. 1) is used to generate the set of regression vectors $\varphi_{j} \quad\left(\varphi_{j}=\right.$ $\left.[y(j) y(j-1) u(j-1)]^{\mathrm{T}}\right)$ for the extended classification procedure to generate the regression vectors cluster centers $\varphi_{i}^{*}$. It generates three clusters centered on the following regression vectors:

$\varphi_{1}^{*}=\left[\begin{array}{l}-0.8149 \\ -0.8181 \\ -0.6874\end{array}\right], \quad \varphi_{2}^{*}=\left[\begin{array}{l}-0.0555 \\ -0.0557 \\ -0.0376\end{array}\right], \quad \varphi_{3}^{*}=\left[\begin{array}{l}0.4655 \\ 0.4646 \\ 0.4369\end{array}\right]$.

For the decision variable $\xi(k)$, we consider the input $u(k)$ (the third element of the regression vector). So, we have the weighting functions centers $c_{i}$ as

$c_{1}=u_{1}^{*}=-0.6874, c_{2}=u_{2}^{*}=-0.0376, c_{3}=u_{3}^{*}=0.4369$.

Using (23), we deduce the corresponding dispersions $\sigma_{i}$ as

$$
\sigma_{1}=0.6498, \quad \sigma_{2}=0.4745, \quad \sigma_{3}=0.4745 .
$$

Fig. 4 gives the resulting weighting functions evolution.

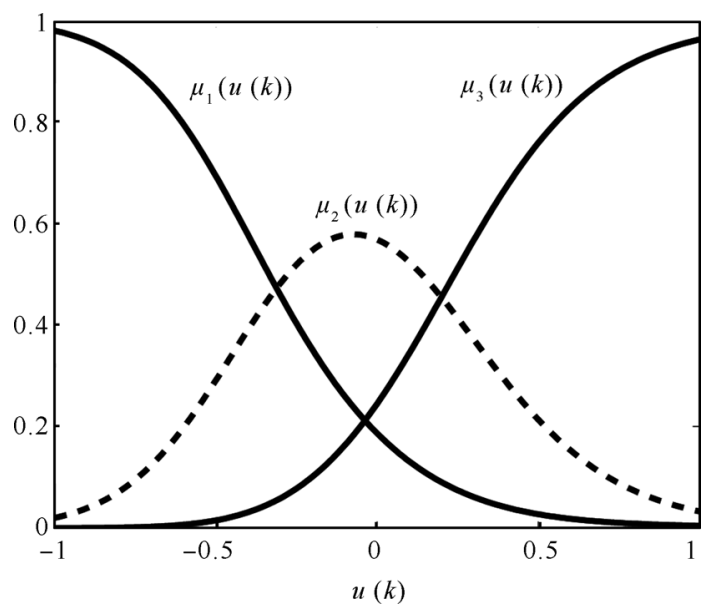

Fig. 4 Weighting functions evolutions

The multimodel identification is realized with a global criterion (as described previously in the identification procedure) and it adopts the uncoupled state multimodel structure. The resulting sub-models are described in the state space by the following expressions:

Sub-model 1.

$$
A_{1}=-0.1468, \quad B_{1}=1.6142, \quad C_{1}=1 .
$$

Sub-model 2.

$$
\begin{aligned}
& A_{2}=\left[\begin{array}{cc}
-0.6938 & -0.3287 \\
-0.14 & 0.9
\end{array}\right], \quad B_{2}=\left[\begin{array}{c}
-2.1141 \\
-0.67
\end{array}\right], \\
& C_{3}=\left[\begin{array}{ll}
1 & 0
\end{array}\right] .
\end{aligned}
$$

Sub-model 3.

$$
A_{3}=0.5334, \quad B_{3}=0.6489, \quad C_{3}=1 .
$$

The same validation conditions are conserved. Fig. 5 illustrates the multimodel validation results. It gives the evolutions of the multimodel response and the real one. We can observe that the multimodel can describe properly the 
non linear system behavior. The MSE and the varianceaccounted-for VAF have been calculated to evaluate the performance of the proposed method. They are respectively equal to 0.003 and $98.81 \%$.

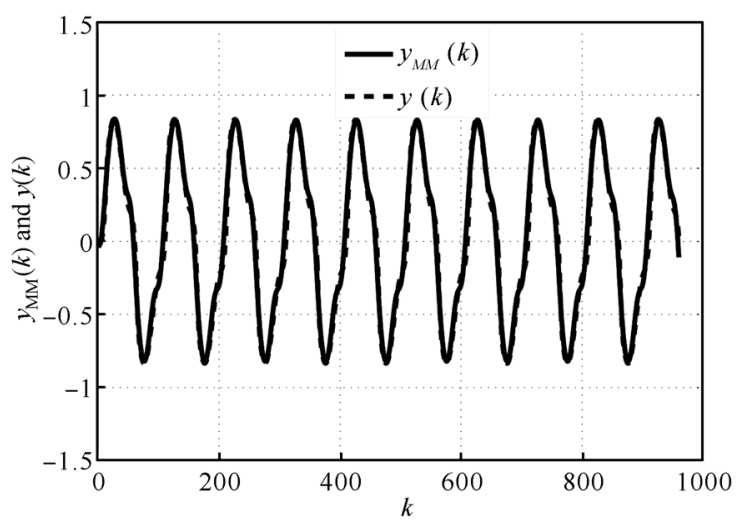

Fig. 5 Evolutions of the multimodel output $y_{M M}(k)$ and the real output $y(k)$. (Systematic and optimal choice of centers and dispersions)

The obtained results using proposed procedure are very good relatively to the case where the classical procedure is used. The following table (Table 1) summarizes the MSE and VAF calculated in the two cases.

Table 1 MSE and VAF

\begin{tabular}{ccc}
\hline & Classical choice & Optimal choice \\
\hline MSE & 0.021 & 0.003 \\
VAF & $92.17 \%$ & $98.81 \%$ \\
\hline
\end{tabular}

\section{Experimental validation}

The satisfactory modeling performances obtained in numerical simulations, incited us to perform an experimental validation on a semi-batch reactor ${ }^{[22,30,31]}$. The plant consists of a perfectly stirred tank provided with a jacket where the heat is exchanged between a cooling fluid and the reaction mixture as shown in Fig. 6. The heating-cooling system fluid flow rate through the jacket is constant and the fluid temperature into the jacket is modulated using an external servo system involving a plate heat exchanger together with electric resistors. The plate exchanger is used for the fluid cooling, namely the tap water, while the heating of the fluid is ensured by the electric resistors. Several temperature sensors allow to measure the reactor temperatures and the inlet and outlet jacked temperatures. The reactor is mainly used in bach mode for chemical esterification of the crude olive oil according to the following reaction:

$$
\text { Acid }+ \text { Alcohol } \rightleftharpoons \text { Ester }+ \text { Water. }
$$

The typical temperature profile for the involved esterification experiment is the following: The reaction mixture is first heated from the room temperature to a specified temperature which usually corresponds to the reaction temperature, then this temperature is maintained constant until the end of the reaction. Finally, the reactor is cooled back to the room temperature.

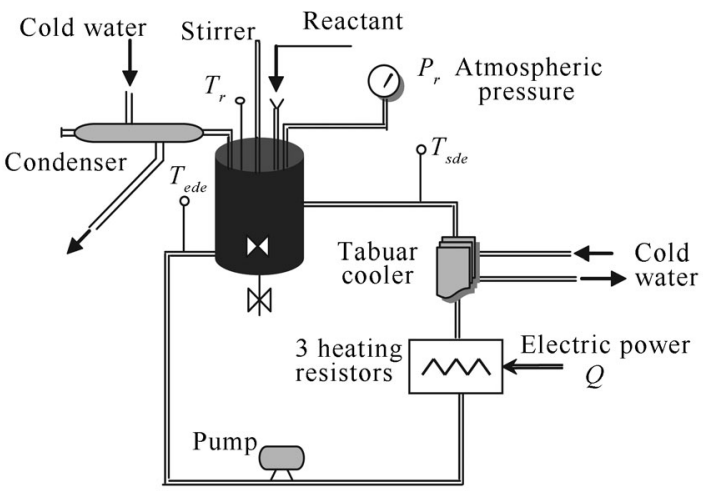

Fig. 6 The chemical reactor

The control variable is the electric power supplied by the heating resistors while the output is the reactor temperature. Such a system is nonlinear as mentioned in $[13,22,32]$. This is the rational behind the multimodel approach to be adopted.

Fig. 7 gives implementation scheme of models' base optimal systematic generation for the considered chemical reactor. The set of identification data picked out of the reactor given by Fig. 8 (the process sampling time is $180 \mathrm{~s}$ ) is treated off line by the classification procedure to generate the weighting functions centers. So, we obtain the following regression vectors centers:

$$
\varphi_{1}^{*}=\left[\begin{array}{c}
83 \\
83 \\
1000
\end{array}\right], \quad \varphi_{2}^{*}=\left[\begin{array}{c}
89 \\
88.1 \\
1700
\end{array}\right], \quad \varphi_{3}^{*}=\left[\begin{array}{c}
104.5 \\
104.4 \\
2000
\end{array}\right] .
$$

When we choose the input $u(k)$ as decision variable, we can deduce the weighting functions centers.

$$
c_{1}=u_{1}^{*}=1000, \quad c_{2}=u_{2}^{*}=1700, \quad c_{3}=u_{3}^{*}=2000 .
$$

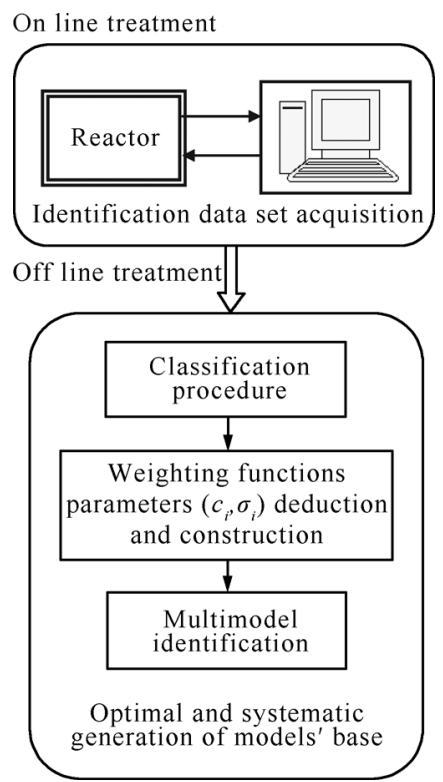

Fig. 7 Implementation scheme of the proposed method for the chemical reactor 

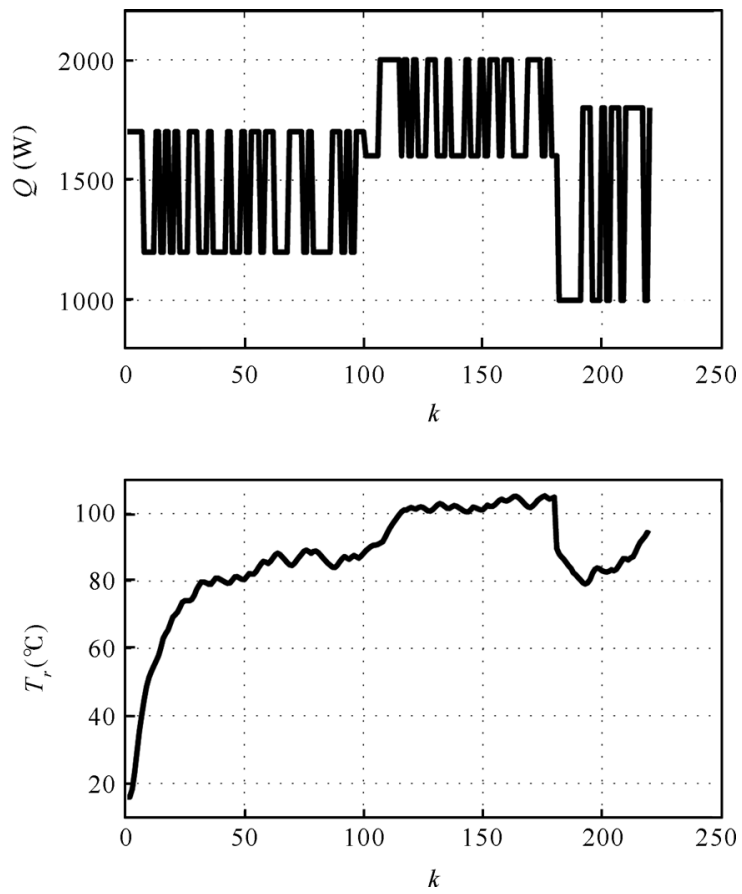

Fig. 8 Identification data set

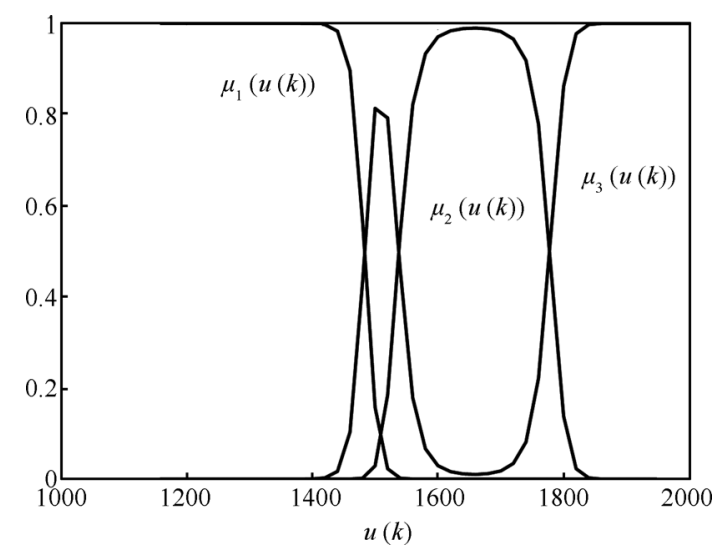

Fig. 9 Evolutions of weighting functions

We deduce the corresponding dispersions using relation $(22)$.

$$
\sigma_{1}=0.0700, \quad \sigma_{2}=0.0263, \quad \sigma_{3}=0.0750 .
$$

Fig. 9 gives the evolution of the resulting weighting functions. The identification procedure application yields to the three sub-models described in the state space as follows:

Sub-model 1.

$$
\begin{aligned}
& A_{1}=\left[\begin{array}{ll}
0.3357 & 0.4002 \\
0.3495 & 0.6685
\end{array}\right], \quad B_{1}=\left[\begin{array}{l}
0.1200 \\
0.1487
\end{array}\right], \\
& C_{1}=\left[\begin{array}{ll}
1 & 0
\end{array}\right] .
\end{aligned}
$$

\section{Sub-model 2}

$$
\begin{aligned}
& A_{2}=\left[\begin{array}{ll}
0.0948 & 0.3740 \\
0.3598 & 0.7085
\end{array}\right], \quad B_{2}=\left[\begin{array}{l}
0.0670 \\
0.3847
\end{array}\right], \\
& C_{2}=\left[\begin{array}{ll}
1 & 0
\end{array}\right] .
\end{aligned}
$$

\section{Sub-model 3.}

$$
\begin{aligned}
A_{3} & =\left[\begin{array}{ll}
0.2279 & 0.3667 \\
0.3500 & 0.7288
\end{array}\right], \quad B_{3}=\left[\begin{array}{l}
0.1819 \\
0.1039
\end{array}\right], \\
C_{3} & =\left[\begin{array}{ll}
1 & 0
\end{array}\right] .
\end{aligned}
$$

The result of the validation phase is given by Fig. 10 which illustrates the evolution of the multimodel output and the reactor one. We can note that the multimodel output describes the real behavior with satisfactory precision. The variance-accounted-for VAF has been calculated to evaluate the performance of the proposed method. It is equal, in this real case, to $98.7 \%$. Fig. 11 shows the relative errors $\operatorname{er}_{M M}(\%)$ (between the real output $y(k)$ and the multimodel output $\left.y_{M M}(k)\right)$. In the full operating regimes (heating, reaction and cooling), the considered error does not exceed $10 \%$.

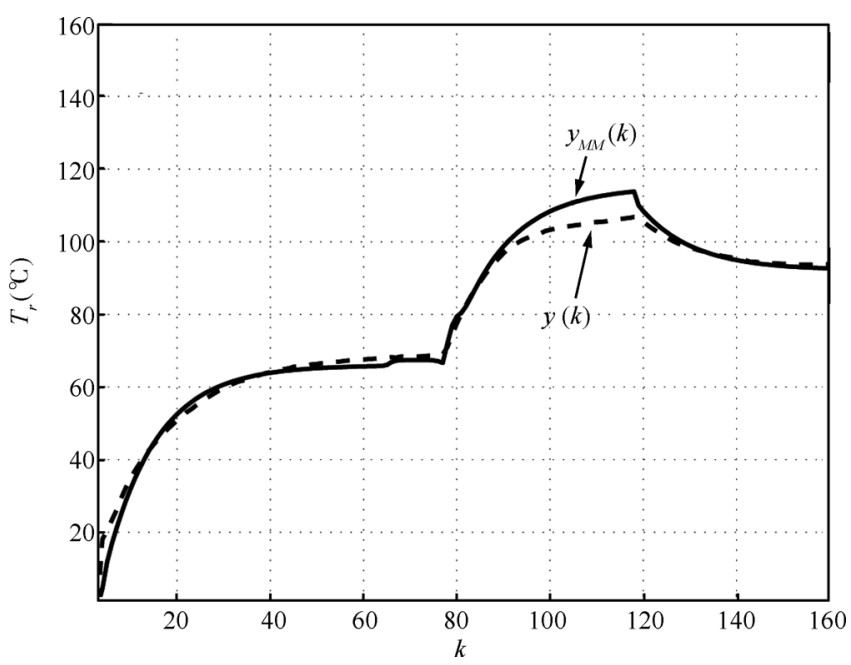

Fig. 10 Evolutions of the multimodel output $y_{M M}(k)$ and the reactor output $y(k)$

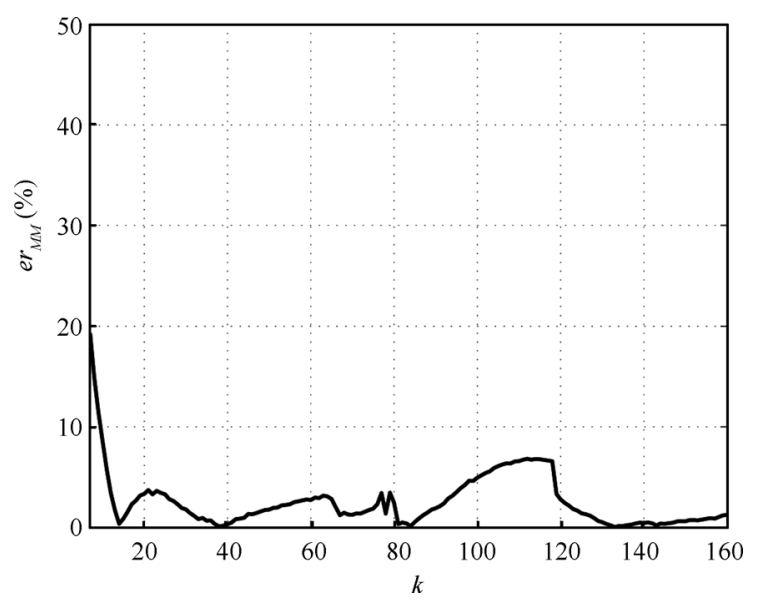

Fig. 11 Evolution of the relative error

\section{Conclusions}

In this paper, we proposed an optimal systematic generation of the weighting function parameters and the models ${ }^{\prime}$ 
base for an uncoupled state multimodel structure. The proposed method integrates a classification procedure of an identification data set picked out of the considered nonlinear system. The obtained results in numerical simulation are very satisfactory relatively to the case where the system static characteristic is exploited. An experimental validation on a chemical reactor is also judged very satisfactory.

\section{References}

[1] R. Babuska, H. B. Verbruggen. Fuzzy set methods for local modelling and identification. Multiple Model Approaches to Modelling and Control, R. Marry-Smith, T. A. Johansen, Eds., London: Taylor and Francis, 1997.

[2] R. Ben Abdennour, P. Borne, M. Ksouri, F. M'sahli. Identification et Commande Numérique Des Procédés Industriels, Paris, France: Technip, 2001. (In Franch)

[3] T. A. Johansen. Operating Regime Based Process Modelling and Identification, Ph. D. dissertation, Norwegian Institute of Technology, Trondheim, Norway, 1994.

[4] T. A. Johansen, B. A. Foss. Operating regime based process modeling and identification. Computers and Chemical Engineering, vol. 21, no. 2, pp. 159-176, 1997.

[5] R. Schorten, R. Murry-Smith, R. Bjorgan, H. Gollee. On the interpretation of local models in blended multiple model structures. International Journal of Control, vol. 72, no. 78, pp. 620-628, 1999.

[6] M. Chadli, M. Didier, J. Ragot. Stability analysis and design for continuous-time Takagi-Sugeno control systems. International Journal of Fuzzy Systems, vol. 7, no. 3, pp. 101109,2005 .

[7] T. Takagi, M. Sugeno. Fuzzy identification of systems and its applications to modeling and control. IEEE Transactions on Systems, Man and Cybernetics, vol. 15, no. 1, 116-132, 1985.

[8] F. Delmotte, L. Dubois, P. Borne. A general scheme for multi-model controller using trust. Mathematics and Computers in Simulation, vol. 41, no. 1-2, pp. 173-186, 1996.

[9] M. Ltaief, K. Abderrahim, R. Ben Abdennour, M. Ksouri. A fuzzy fusion strategy for the multimodel approach application. Wseas Transactions on Circuits and Systems, vol. 2, no. 4, pp. 686-691, 2003.

[10] S. Mezghani, A. Elkamel, P. Borne. Multimodel control of discrete systems with uncertainties. International Journal of Studies in Informatics and Control, vol. 3, no. 2, pp. 7-17, 2001.
[11] S. Talmoudi, R. Ben Abdennour, K. Abderrahim, M. Ksouri. A New technique of validities' computation for multimodel approach. Wseas Transactions on Circuits and Systems, vol. 2, no. 4, pp. 680-685, 2003.

[12] S. Talmoudi, K. Abderrahim, R. Ben Abdennour, M. Ksouri. Multimodel approach using neural networks for complex systems modeling and identification. Journal of Nonlinear Dynamics and Systems, vol. 8, no. 3, pp. 299-316, 2008.

[13] M. Ltaief, K. Abderrahim, R. Ben Abdennour, M. Ksouri. Contributions to the multimodel approach: Systematic determination of a models' ${ }^{\prime}$ base and validities estimation. International Journal of Automation and Systems Engineering, vol. 2, no. 3, 2008.

[14] D. Filev. Fuzzy modeling of complex systems. International Journal of Approximate Reasoning, vol. 5, no. 3, pp. 281290, 1991

[15] K. Tanaka, M. Sugeno. Stability analysis and design of fuzzy control systems. Fuzzy Sets and Systems, vol. 45, no. 2, pp. 135-156, 1992.

[16] T. M. Guerra, A. Kruszewski, L. Vermeiren, H. Tirmant. Conditions of output stabilization for nonlinear models in the Takagi-Sugeno's form. Fuzzy Sets and Systems, vol. 157, no. 9 , pp. $1248-1259,2006$

[17] Ragot J, Marx B, Koenig D. Design of observers for TakagiSugeno descriptor systems with unknown inputs and application to fault diagnosis. IET Control Theory and Applications, vol. 1, no. 5, pp. 1487-1495, 2007.

[18] F. Ahmida, E. H. Tissir. Exponential stability of uncertain T-S fuzzy switched systems with time delay. International Journal of Automation and Computing, vol. 10, no. 1 , pp. 32-38, 2013

[19] R. Orjuela, D. Maquin, J. Ragot. Nonlinear system identification using uncoupled state multiple-model approach. In Proceedings of Workshop on Advanced Control and Diagnosis, Nancy, France, 2006.

[20] R. Orjuela, B. Marx, J. Ragot, D. Maquin. State estimation of nonlinear discrete-time systems based on the decoupled multiple model approach. In Proceedings of the 4th International Conference on Informatics in Control, Automation and Robotics, Angers, France, 2007.

[21] R. Orjuela, B. Marx, D. Maquin, J. Ragot. A decoupled multiple model approach for state estimation of nonlinear systems subject to delayed measurements. In Proceedings of the 3rd IFAC Advanced Fuzzy and Neural Network Workshop, IFAC, Valenciennes, France, pp. 29-30, 2008. 
[22] A. Messaoud, M. Ltaief, R. Ben Abdennour. A new contribution of an uncoupled state multimodel predictive control: Experimental validation on a chemical reactor. International Review of Automatic Control, vol. 3, no. 5, pp. 550$559,2010$.

[23] A. Messaoud, M. Ltaief, R. Ben Abdennour. Partial predictors for the supervision of a multimodel direct generalized predictive control. In Proceedings of the American Control Conference, IEEE, Seattle, USA, pp. 459-464, 2008.

[24] R. Murray-Smith, T. Johansen. Multiple Model Approaches to Modelling and Control, London: Taylor \& Francis, 1997.

[25] R. Orjuela. Contribution l'estimation d'état et au diagnostic des systés représentés par des multimodèles, Ph. D. dissertation, Institut National Polytechnique de Lorraine, Nancy, France, 2008. (In Franch)

[26] S. Bedoui, M. Ltaief, K. Abderrahim. New results on discrete-time delay systems identification. International Journal of Automation and Computing, vol.9, no. 6, pp. 570-577, 2012.

[27] S. L. Chiu. Fuzzy model identification based on cluster estimation. Journal of Intelligent and Fuzzy Systems, vol. 2, no. 3, pp. 267-278, 1994.

[28] S. Abe. Neural Networks and Fuzzy Systems: Theory and Applications, Boston: Kluwer Academic Publishers, 1997.

[29] K. Narendra, K. Parthasarathy. Identification and control of dynamical systems using neural networks. IEEE Transactions on Neural Networks, vol. 1, no. 1, pp. 4-27, 1990.

[30] F. M'sahli, C. Fayeche, R. Ben. Abdennour, M. Ksouri. Application of adaptive controllers for the temperature control of a semi-batch reactor. International Journal of Computational Engineering Science, vol. 2, no. 2, pp. 287-307, 2001.

[31] F. M'sahli, R. Ben. Abdennour, M. Ksouri. Experimental nonlinear model based predictive Control For a Class of semi-bath reactor. International Journal of Advanced Manufacturing Technology, vol. 20, no. 6, pp. 459-463, 2002.

[32] A. Messaoud, M. Ltaief, R. Ben. Abdennour. Supervision based on partial predictors for a multimodel generalized predictive control: Experimental validation on a semi-batch reactor. International Journal of Modelling, Identification and Control, vol. 6, no. 4, pp. 333-340, 2009.

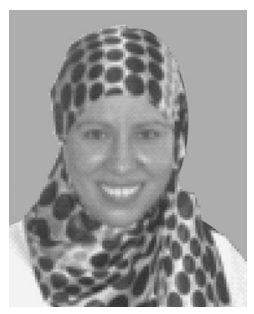

Majda Ltaief received her engineering diploma in electrical-automatic from $\mathrm{Na}$ tional School of Engineers of Gabes-Tunisia in 1996 and the master degree from the National School of Engineers of Tunis-Tunisia in 1999. In 2005, she obtained her Ph. D. degree in electrical engineering from National School of Engineers of Tunis-Tunisia. In 2013, she obtained her Habilitation thesis to supervise research in electrical engineering from the University of Gabes-Tunisia. She is currently a professor in the Electric Engineering Department of National School of Engineers of Gabes-Tunisia.

Her research interests include multimodel and multicontrol approaches, fuzzy supervision, and numerical control of complex systems.

E-mail: majda.ltaief@enig.rnu.tn (Corresponding author)

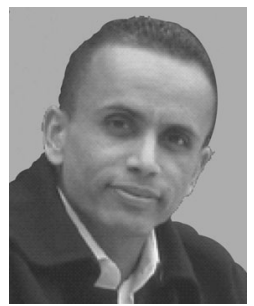

Anis Messaoud received the engineering and master degrees in electrical engineering and automatic control from $\mathrm{Na}$ tional School of Engineers of GabesTunisia, in 2004 and 2006, respectively. In 2010, he obtained his $\mathrm{Ph}$. D. degree in electrical-automatic engineering from the National School of Engineers of Gabes, Tunisia. He is currently an associate professor in the Electric Engineering Department in National School of Engineers of Gabes-Tunisia.

His research interests include predictive control of complex systems, multimodel and multicontrol approaches.

E-mail: messaoud_anis@yahoo.fr

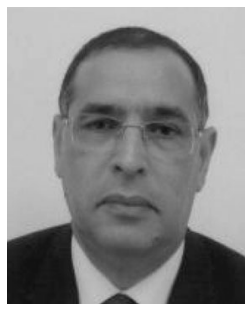

Ridha Ben Abdennour received the Doctorat de spécialité degree from the Ecole Normale Supérieure de $\mathrm{l}^{\prime}$ Enseignement Technique in 1987 , and the Doctorat $d^{\prime}$ Etat degree from the Ecole Nationale d'Ingénieurs de Tunis in 1996. He is a professor in automatic control at the National School of Engineers of Gabes-Tunisia. He was chairman of the Electrical Engineering Department and the director of the High Institute of Technological Studies of Gabes. He is the head of the Research Unit of Numerical Control of Industrial Processes and is the founder and honorary president of the Tunisian Association of Automatic and Numerisation. $\mathrm{He}$ is the co-author of the book of Identification and Numerical Control of Industrial Processes and he is the author of more than 300 publications. He participated in the organization of many conferences and he was member of some scientific congress committees.

His research interests include identification, multimodel \& multicontrol approaches, numerical control and supervision of industrial processes.

E-mail: ridha.benabdennour@enig.rnu.tn 